\title{
SEM and STEM Microstructural and Microchemical Analysis of Pigments Contained in a Salvator Mundi in the Collection of the Detroit Institute of Arts.
}

\author{
John F. Mansfield ${ }^{1}$, Brenda M. Vyletel ${ }^{2}$ and Cathy Selvius DeRoo ${ }^{3}$
}

1. \& 2. North Campus Electron Microbeam Analysis Laboratory, 417 SRB, University of Michigan, 2455 Hayward, Ann Arbor MI 48109-2143 USA

\section{Detroit Institute of Arts, 5200 Woodward Avenue, Detroit, Michigan 48202, USA}

A number of paintings entitled Salvator Mundi (Savior of the World), depict Christ with his right hand raised in blessing and his left hand holding an orb representing the universe [1]. A recently rediscovered Salvator Mundi, which has received substantive scholarly scrutiny and conservation treatment to remove crude overpaint, is now accepted as painted by Leonardo da Vinci and was exhibited at the National Gallery London in 2012 (Figure 1a). The Leonardo Salvator Mundi once resided in the collection of King Charles I of England. The Salvator Mundi paintings from the Detroit Institute of Arts (Figure 1b) and from the Parisian collection of Jean Louis de Ganay [2] (Figure 1c) are copies of the Leonardo painting by unknown artists. The DIA Salvator Mundi was included in the 2010-11 exhibition entitled "Fakes, Forgeries, and Mysteries". The DIA painting has been in the collection since the late $19^{\text {th }}$ century: its date of attribution and the artist's identity are unknown.

To refine the date of attribution of the DIA Salvator Mundi, non-destructive X-ray fluorescence (XRF) analysis was performed initially and followed by analysis of microscopic paint samples extracted for characterization by optical microscopy, scanning electron microscopy (SEM), and X-ray energy dispersive spectrometry (XEDS). Transmission electron microscope sections were also extracted in focused ion beam (FIB) workstation and characterized by scanning transmission electron microscopy (STEM) and XEDS.

XRF performed at the DIA, revealed that the yellow highlights on the gown embroidery (Figure 2a.) contain tin and lead, indicating the use of lead tin yellow, a synthetic yellow pigment that fell out of use by about the mid-18th century. Optical microscopy of mounted samples under UV illumination revealed sharp angular shards that varied in color from blue to bluish gray to black. XRF analysis of the areas from which the samples were removed detected the presence of cobalt, arsenic, nickel, and traces of bismuth in addition to $\mathrm{K}$ and $\mathrm{Si}$, indicative of the use of smalt, a blue ground glass used as a pigment. SEM revealed that the very dark shards were carbon and were probably graphitic in nature. Other shards, examined by SEM, XEDS and, after FIB liftout, (S)TEM and XEDS, were identified as smalt, which was prevalent in easel paintings during the $15-17^{\text {th }}$ centuries The blue color of the smalt turns to a gray hue over time [3], as the potassium migrates out of the glass (Figure 3). Smalt became far less popular in the 18th century when Prussian blue (hexacyanoferrate(II) ) pigments became available [4]. The detection of bismuth in the dark blue areas of the painting raised questions of its role in the painting. Bismuth is known to have been used in the $16^{\text {th }}$ century as a black pigment, however, the level of bismuth was never detected in XRF, SEM XEDS or TEM XEDS at any level above a trace and was found to be uniformly distributed wherever it was observed, hence it is believed to be present as a constituent of the original cobalt ore. 
The use of PbSn yellow and smalt strongly suggest that the painting predates the mid $18^{\text {th }}$ century. Analysis continues, particularly of the fine phases in STEM.

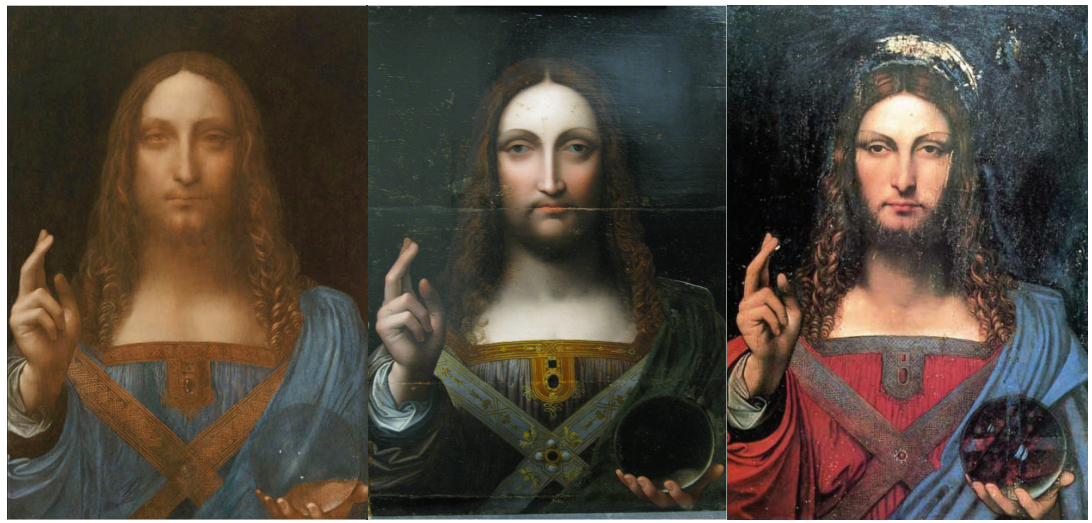

Figure 1. Three examples of Salvator Mundi: a. the recently indentified version by Leonardo da Vinci from a private collection, $b$. from the Detroit Institute of Arts collection, and c. from the collection of Jean Louis de Ganay[2]

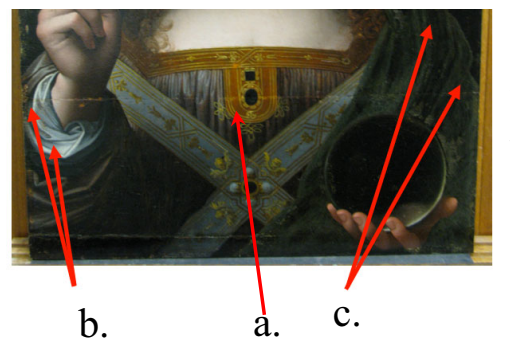

Figure 2. Locations where paint samples were extracted for microchemical and microstructural analysis. a. Lead tin yellow/ yellow ochre detail on the gown edging. b. light blue area of gown sleeve and c. very dark blue of the stole.
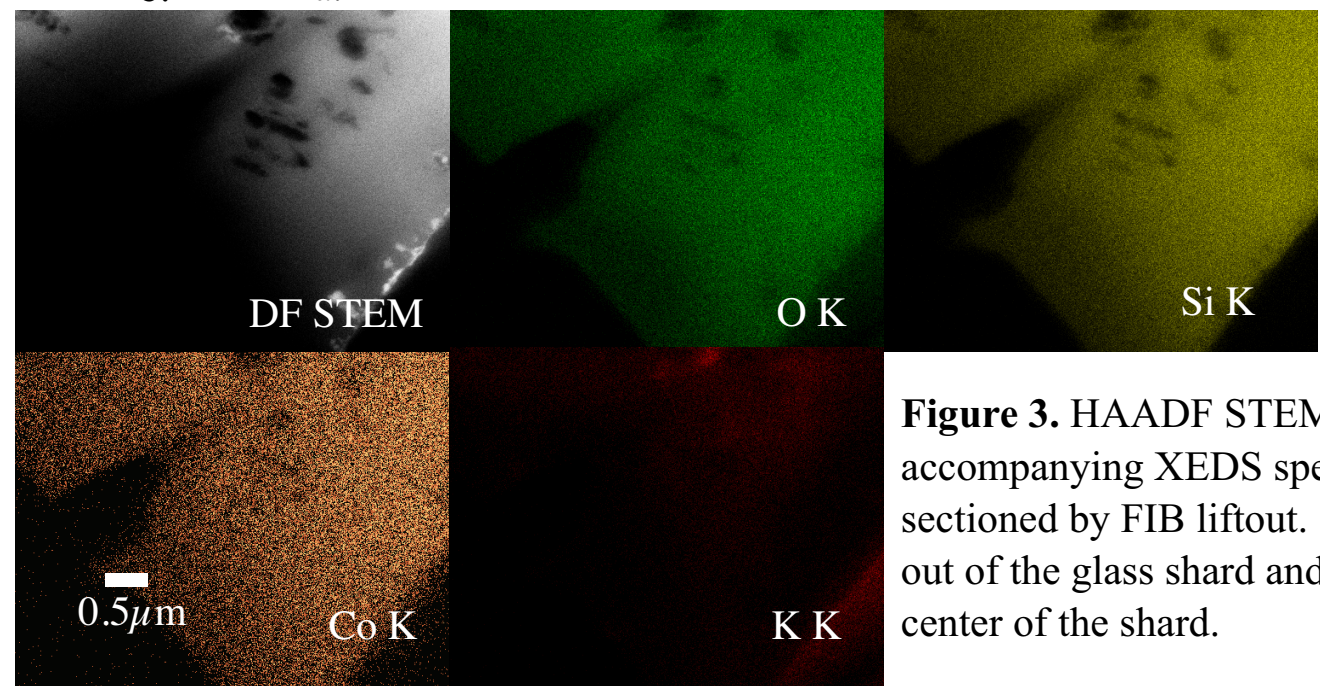

Figure 3. HAADF STEM image and accompanying XEDS spectrum maps of a shard sectioned by FIB liftout. Potassium has migrated out of the glass shard and only remains in the very center of the shard.

\section{References}

[1] Joanne Snow-Smith, "The Salvator Mundi of Leonardo Da Vinci”, (Henry Art Gallery, University of Washington, USA) 1982.

[2] http://www.lairweb.org.nz/leonardo/mundi.html

[3] Spring, M., Higgitt, C., Saunders, D. "Investigation of Pigment-Medium Interaction Processes in Oil Paint containing Degraded Smalt”. National Gallery Technical Bulletin Vol 26, pp 56-70.

http://www.nationalgallery.org.uk/technical-bulletin/spring_higgitt_saunders2005

[4] N. Eastaugh, et al, "Pigment Compendium - A Dictionary and Optical Microscopy of Historical Pigments (Butterworth-Heinemann, Burlington, MA, USA). 